\title{
El fundamento lingüístico del pensamiento de Adam Smith.
}

\section{The Linguistic Foundation of Adam Smith's Thought.}

RECIBIDO: 13 DE FEBRERO DE 2018 / ACEPTADO: 20 DE OCTUBRE 2018

\section{JORGE LÓPEZ LLORET}

Universidad de Sevilla. lopezlloret@us.es

Resumen: En la reconstrucción del sistema filosófico de Adam Smith, sus estudios sobre el lenguaje son una pieza clave en varios sentidos: establecen la necesidad como la base del desarrollo de la sociedad humana; definen la pauta histórica que sigue dicho desarrollo; identifican la fórmula fundamental según la cual tienen lugar las interacciones sociales; establecen el paso de una conciencia aislada a otra dialógica; y muestran la estructura teatral de las relaciones sociales. Todo esto permite abordar una interpretación de su obra no como una estructura económica determinista, sino como una construcción semiótica a la que podríamos dar múltiples formas.

Palabras clave: Lenguaje, Historia, Desarrollo, Ética, Economía.
Abstract: In the reconstruction of Adam Smith's philosophical system, his studies on language are a key piece in several senses: they establish the necessity as the base of the development of the human society; define the historical pattern that this development follows; identify the fundamental formula according to which social interactions take place; establish the passage from an isolated consciousness to a dialogical one; and evince the theatrical structure of social relations. All this allows us to develop an interpretation of his work not as a deterministic economic structure, but as a semiotic construction to which we could give multiple forms.

Keywords: Language, History, Development, Ethics, Economy. 


\section{INTRODUCCIÓN.}

Consideraciones sobre la formación original de los lenguajes es, aparte de La Teoría de los sentimientos morales (1759) y La riqueza de las naciones (1776), la única obra firmada que Smith publicó en vida. Sus otras publicaciones fueron anónimas, póstumas o apuntes de asistentes a sus clases. Dada su importancia, resulta llamativo el escaso interés que ha despertado entre el público castellano, lo mismo que las aún no traducidas Lecciones sobre retórica, de las cuales la tercera recoge un resumen ${ }^{1}$. Este escaso interés es problemático, pues Consideraciones está llamada a jugar un papel importante como núcleo integrador en el proceso contemporáneo de recuperación de la base filosófica de la obra de Smith, ensombrecida por sus valores económicos.

Adam Smith publicó Consideraciones en 1761, adjuntándola en 1767 a la tercera edición de La teoría de los sentimientos morales. Un mal anfitrión según Plank, opinión compartida por Swearingen, para quien Smith, con su racionalismo taxonómico, concedió poca atención al lenguaje como condición necesaria de la sociedad humana ${ }^{2}$. Para nosotros esto es incorrecto porque interpreta Consideraciones descontextualizándola del resto de la obra de Smith. A partir del contexto global de su producción como escritor y profesor, se pueden alcanzar conclusiones diferentes, como hicieron McKenna y Otteson. Según el primero, para Smith el estudio del lenguaje era previo al de la ética, mientras que para el segundo Consideraciones afinó el método filosófico desarrollado en el resto de su obra ${ }^{3}$.

Creemos que Consideraciones es relevante para interpretar de una manera más integral la obra de Smith al menos por tres motivos: en primer lugar, porque conecta las Lecciones sobre retórica con La teoría de los sentimientos morales, a través de la identificación de la conciencia dialógica como la base de la comunicación y del juicio moral; en segundo lugar, porque aclara la base lingüística de la fórmula del intercambio, importante a partir de las Lecciones sobre jurisprudencia, y define la estructura básica de su desarrollo histórico, importante en La riqueza de las naciones; $\mathrm{y}$, en tercer lugar, porque ayuda a establecer más sólidamente las relaciones entre la economía y la ética a través de la ley de los

1 Recientemente han aparecido dos traducciones de Consideraciones, la primera de Carrión, G. en Smith, A. (2017), pp. 101-31 y la segunda de López Lloret, J. en Smith, A. (2018), pp. 37-101. Usaremos la última.

2 Plank, F. (1992), p. 22; Swearingen, C. J. (2013), p. 169.

3 McKenna, S. J. (2006), p. 78; Otteson, J. R. (2013), p. 15. 
cuatro estadios de desarrollo social, integrando con ello planteamientos de $L a$ teoría de los sentimientos morales y de La riqueza de las naciones.

Para mostrar eso hemos identificado y analizado, en lo que sigue, cuatro temas fundamentales presentes en Consideraciones: primero, la necesidad y la importancia de la ayuda mutua, base histórica de toda socialización; segundo, la fórmula básica de toda interacción humana, que adopta la forma "dame aquello que necesito y tendrás esto que necesitas"; tercero, la hipótesis del desarro$l l o$ desde una síntesis inarticulada hacia un análisis articulado, que le proporcionó un esquema básico en el que ubicar todos los procesos históricos; y, cuarto, las condiciones para el surgimiento de una conciencia dialógica y una comunicación teatralizada, de las que surgió una relación humana basada en la simpatía.

Como conclusión, veremos que la interpretación de la obra de Smith a partir de Consideraciones realza la condición semiótica y artificial del ser humano en tanto que ser social (puesto que el lenguaje es algo construido por nosotros y, según Smith, la sociedad solo es posible por el lenguaje), su papel activo en la construcción de una vida cada vez menos sometida a las restricciones naturales y, por eso mismo, a las leyes de un mercado identificado con el sistema del mundo newtoniano. La propia naturaleza abierta del método de investigación de Newton, a lo que Smith, según mostró Leónidas Montes ${ }^{4}$, fue sensible gracias, seguramente, a las objeciones que su amigo Hume opuso a la posibilidad de la certeza en las cuestiones de hecho (a diferencia de lo que sucedía con las relaciones entre ideas ${ }^{5}$ ), le permitían adoptar esta posición a la vez creativa y legalista, según la cual el mercado lo produce el ser humano porque es un animal semiótico, de manera que, para bien o para mal, se hace el autor de lo que llega a ser y, con ello, más responsable ante sí mismo y ante la naturaleza que tutela.

\section{LA OMNIPRESENCIA DE LA NECESIDAD.}

Tras asistir a la tercera de las Lecciones sobre retórica, el anónimo oyente de Smith anotó que "dos salvajes que se encontraron y pasaron a residir en el mismo lugar muy pronto se procuraron signos para denotar los objetos más

4 Montes Lira, L. (2009), pp. 140-151.
5 Hume, D. (2015), pp. 64-82. 
frecuentes y que más les interesaban"6. Esos objetos fueron la cueva que habitaron, el árbol que los alimentó o la fuente de la que bebieron, todos ellos de importancia vital. El oyente que anotó esto, como algunos analistas posteriores de Consideraciones, interpretó la teoría del lenguaje de Smith en términos denotativos ${ }^{7}$. Sin embargo, Consideraciones aporta añadidos notables. Por un lado, se trata de salvajes "criados lejos de las sociedades humanas y a los que nunca se ha enseñado a hablar", introduciéndose con ello la conjetura roussoniana del primitivo aislado y solitario; por otro lado, lo que buscaban era "hacer inteligibles el uno al otro sus necesidades comunes, pronunciando ciertos sonidos siempre que pretendieran denotar ciertos objetos". Sus signos, pues, no denotaron inmediatamente objetos sino necesidades, las cuales no son un estado del mundo sino una situación del sujeto?.

Smith no creyó, en realidad, en la existencia humana del primitivo aislado, pues sin lenguaje, que es nuestra característica distintiva, no había humanidad $^{10}$. El lenguaje surgió como un recurso para expresar una situación carencial y conseguir ayuda a través de la reciprocidad, constituyéndose como el instrumento fundamental del proceso socializador. Este recurso humano surgió de nuestra necesidad de dirigir la conducta ajena para lograr ayuda, pues los humanos, como afirmó Smith en sus Lecturas sobre jurisprudencia, estamos por naturaleza "en una condición mucho más desvalida y necesitada con respecto al mantenimiento y la comodidad" de nuestra vida que el resto de los animales, encontrando "que en todo hace falta mejora" 11 . Sobre esta base, presentó el desarrollo histórico desde las necesidades hasta las superficialidades como algo que respondía al esfuerzo por minimizar las primeras, incluso en culturas tan sofisticadas como la británica o la francesa de mediados del siglo XVIII, de manera que "todas las artes se han inventado y mejorado para suplir las necesidades de comer, beber, vestirse y alojarse" (Smith incluye entre estas artes las ciencias, el derecho, el gobierno, la sabiduría e, incluso, la virtud) ${ }^{12}$.

6 "Two Savages who met together and took up their dwelling in the same place would very soon endeavour to get signs to denote those objects which most frequently occurred and with they were most concerned"; Smith, A. (1985), p. 9. Traducción nuestra.

7 Land, S. K. (1977), p. 680; Land, S. K. (1986), p. 142.

8 Smith, A. (2018), p. 39; Smith, A. (1985), p. 203.

9 Christie, J. R. R. (1987), p. 208; Conlin, J. (2016), p. 58.

10 Smith, A. (1997), pp. 586-7; Smith, A. (1982a), p. 336.

11 Smith, A. (1995), pp. 380-381; Smith, A. (1982c), pp. 334-335; Smith, A, (1996), p. 133; Smith, A. (1982c), p. 487.

12 Smith, A. (1995), pp. 383 y 385; Smith, A. (1982c), pp. 337 y 338. 
"Cueva", "árbol” y "fuente” seguían siendo, pues, las palabras fundamentales incluso en las sofisticadas sociedades comerciales ${ }^{13}$.

Estas reflexiones, que se hallaban justo antes del tratamiento de la división del trabajo en las Lecturas sobre jurisprudencia y que mostraban la naturaleza esencial de la sociabilidad humana, Smith las dio por supuestas en La riqueza de las naciones (en 1776, cuando publicó su magna obra, Consideraciones ya llevaba quince años publicada, de ellos nueve como acompañante de La teoría de los sentimientos morales), pero no en el primer párrafo del Borrador previo de dicha obra, donde anotó que, solo con su trabajo, el individuo no podría conseguir "su alimento, vestido y albergue, no digo para disfrutar de los mayores lujos, sino ni siquiera para satisfacer los apetitos naturales que tendría el campesino más austero en cualquier sociedad civilizada" ${ }^{14}$. La condición antropológica del ser humano, el animal necesitado, era, pues, la vida en sociedad, de manera que la especie humana solo fue viable porque comenzó a comunicar sus necesidades a través del lenguaje, poniendo en práctica una cooperación que, sin anularlas, las satisfacía. Consideraciones, por lo tanto, debería leerse como el punto de partida de la obra que más apreciamos de Smith, La riqueza de las naciones, cuyo tema esencial es, en el fondo, la necesidad humana y la manera más rentable y universal de satisfacerla.

\section{LA FÓRMULA BÁSICA DE LA INTERACCIÓN LINGÜÍSTICA.}

La trama económica que Smith analizó tan minuciosamente en La riqueza de las naciones tenía como base una fórmula lingüística utilizada en las Lecciones sobre jurisprudencia y en el Borrador previo. A partir del hecho de que el ser humano, por ser el animal necesitado, debía recurrir a la ayuda de los otros, identificó en las Lecciones una propensión general al trueque y una inclinación "natural" a persuadir a los demás de la conveniencia del intercambio $^{15}$. Con esta situación, que verbalizó en el Borrador como "dame aquello que quiero y tendrás esto que quieres", ${ }^{16}$ nuestro autor abrió Consideraciones, definiéndola en La riqueza de las naciones como "la consecuencia necesaria de las facultades de la razón y el lenguaje" ${ }^{17}$.

13 Phillipson, N. (2010), p. 115.

14 Smith, A. (2017), p. 133; Smith, A. (1982c), p. 562.

15 Smith, A. (1995), pp. 395 y 400; Smith, A. (1982c), pp. 347 y 352.

16 Smith, A. (2017), p. 150; Smith, A. (1982c), p. 571.

17 Smith, A. (1994), p. 44; Smith, A. (1981), p. 25. 
Si verbalizamos el encuentro entre los salvajes al comienzo de Consideraciones obtendremos dicha fórmula, lo mismo que si verbalizamos cada interacción en cada fase del desarrollo social, incluida la comercial ${ }^{18}$. La riqueza de las naciones es, de hecho, una extraordinaria estructura surgida de la multiplicación de esta célula lingüística original. Pensemos en el ejemplo que puso Smith del abrigo de lana de un jornalero, obra colectiva del pastor, el ganadero, el cortador de lana, quien usa unas tijeras producidas entre el minero, el constructor del horno de fundición, el leñador que obtiene la madera de la que surge el carbón, el fabricante de ladrillos, el albañil, el fraguador o el herrero, el clasificador de la lana, el que la recoge, el peinador, el que la tiñe, el hilandero, el tejedor, el batanero, el sastre, el mercader, el transportista, los diseñadores de máquinas como el telar o el molino o los constructores de barcos, cuerdas y velas implicados en el transporte de los tintes ${ }^{19}$.

Tales conexiones podían ampliarse hasta abarcar todo el sistema productivo, al que se podría aplicar la fórmula lingüística básica con independencia de su simplicidad o complejidad. Siguiendo el esquema de evolución de la "ley de los cuatro estadios", al principio es algo simple y personal: un salvaje cazador hace mejores arcos en menos tiempo que otros salvajes, los intercambia ocasionalmente por algún venado y acaba por dedicarse a fabricarlos ${ }^{20}$. En términos verbales, este salvaje diría a otro: "dame el venado que necesito y te daré el arco que necesitas". Después, en las fases más desarrolladas, mediatizadas por el dinero, todo llega a ser más complejo, pero la fórmula se mantiene, pues el dinero solo es el intercambiador universal21 ${ }^{21}$ Por ejemplo, cuando en la confección de paños un fabricante adquiere un tinte a cambio de una suma de dinero, es como si le dijera al comerciante: "dame el tinte que necesito y te daré lo que te permite conseguir lo que necesitas", y así sucesivamente. Aunque la fórmula lingüística surge en la interacción entre individuos, la relación entre Estados también la sigue, pues con ellos sucede, según Smith, lo mismo que con los individuos ${ }^{22}$. Así, por ejemplo, cuando este detectó las principales ventajas del descubrimiento de América y de un pasaje a las Indias Orientales a través del cabo de Buena Esperanza, para él los dos eventos más importantes de la historia de la humanidad, interpretó que esa unión de las dos partes más distantes del mundo les permitía "aliviar sus necesidades recíprocas", lo que

18 Schliesser, E. (2017), p. 43.

19 Smith, A. (2017), p. 133; Smith, A. (1982c), p. 562.

20 Smith, A. (2017), p. 151; Smith, A. (1982c), p. 572.

21 Smith, A. (1994), p. 374; Smith, A. (1981), p. 290.

22 Smith, A. (1994), p. 467; Smith, A. (1981), p. 365. 
casi repetía la afirmación inicial de Consideraciones ${ }^{23}$. La fórmula lingüística básica, pues, fue el principal agente de la globalización socioeconómica en el mundo moderno, extendiéndose a todo el sistema social, que era una inmensa red lingüística basada en la carencia ${ }^{24}$.

El hecho de que nuestra naturaleza carencial nunca se superase, estando presente incluso en la adquisición de lo superfluo, fue una causa fundamental del aumento progresivo del consumo, que requirió y estimuló un proceso creciente de división del trabajo, lo cual nos conduce a la pauta fundamental del desarrollo histórico, que Smith expuso con claridad en Consideraciones.

\section{LA HIPÓTESIS DEL DESARROLLO.}

Consideraciones proporcionó a La riqueza de las naciones la célula básica de toda comunicación, pero también un modelo estructural para la historia humana, pues Smith afirmó que la historia del lenguaje se basaba en decisiones humanas, pero también en que estas decisiones producían consecuencias "sin intención ni previsión por parte de los que establecieron el ejemplo originalmente, quienes nunca trataron de crear una regla general. Esta se establecería por sí misma insensible y gradualmente" ${ }^{25}$. En este capítulo partimos de la interpretación que Otteson, apoyándose en Keller, hizo de este párrafo, en el que detectó el origen de la idea de que los actos humanos tenían consecuencias que eran el producto de la acción, pero no del diseño, humanos ${ }^{26}$. Es parte de la idea más famosa de La riqueza de las naciones, pues, según Otteson, dio lugar a la metáfora de la mano invisible. Este tema de las consecuencias no planificadas de la acción humana está muy presente en Smith, no solo en el sentido positivo característico de la citada metáfora, donde el egoísmo individual genera beneficios colectivos, sino que también le permitió desentrañar aspectos oscuros de los procesos humanos, comenzando con la propia conclusión de Consideraciones, donde el desarrollo analítico de los lenguajes modernos los hizo óptimos para el conocimiento a costa de la libertad estética de los lenguajes antiguos, más plásticos ${ }^{27}$. Esto tuvo una manifestación más dra-

23 Smith, A. (1994), p. 621; Smith, A. (1981), p. 626.

24 Conlin, J. (2016), pp. 110-112.

25 Smith, A. (2018), p. 61; Smith, A. (1985), p. 211.

26 Conlin, J. (2016), pp. 110-112. Smith, A. (2018), p. 61; Smith, A. (1985), p. 211. (2002a), pp. 16, 21-22 y 264-269; Otteson, J. R. (2002b), pp. 77-82; Keller, R. (1994), pp. 35-8.

27 Smith, A. (2018), pp. 96-101; Smith, A. (1985), pp. 224-226. 
mática cuando, en el Libro V de La riqueza de las naciones, Smith opuso a los beneficios de la división del trabajo, presentados en el Libro I, el daño que producía entre los obreros que se subordinaban a ella $^{28}$. La educación y la estética, que tanto interesaron a Smith, podrían paliar estos efectos negativos, lo que se muestra en su defensa de la poesía grecolatina al final de Consideraciones $^{29}$. Fue una vía que siguió después, por ejemplo, Friedrich Schiller en sus Cartas sobre la educación estética de la bumanidad.

Nosotros creemos que el lenguaje le proporcionó a Smith, en cualquier caso, un modelo básico, según el cual la historia discurre desde unas situaciones de homogeneidad no estructurada hacia otras de heterogeneidad muy estructurada. El texto fundamental que soporta esto es una comparación del lenguaje con las máquinas al final de Consideraciones, donde Smith (que por entonces estaba interesado en la historia de los mecanismos, como mostró en sus Lecciones sobre furisprudencia ${ }^{30}$ ), tras indicar que en las máquinas más primitivas cada parte tenía su propia fuente motora y que en las más modernas dichas fuentes se reducían todo lo posible, gracias al desarrollo de formas más sofisticadas y especializadas de transmisión de los movimientos, afirmó que lo mismo sucedía con el lenguaje. Al principio, cada palabra cumplía varias funciones, pero con el tiempo se fueron especializando, de manera que "un conjunto de palabras era capaz de ocupar el lugar de aquel número infinito, de modo que cuatro o cinco preposiciones y media docena de verbos auxiliares podían responder al mismo fin que todas las declinaciones y conjugaciones de los lenguajes antiguos" ${ }^{31}$.

Con respecto a la homogeneidad original no estructurada, según Smith (quien seguía en esta afirmación a Rousseau), los lenguajes antiguos fueron sintéticos porque el salvaje estaba más próximo a la naturaleza, cuyos fenómenos eran realidades globales. Una palabra era entonces como una oración y el conjunto de fenómenos no se diferenciaba en distintas partes componentes. Por ejemplo, según afirma, "en la naturaleza la idea o concepción de Alejandro paseando es tan completa y perfectamente simple como la de Alejandro

28 Smith, A. (1994), p. 717-22 (traducción incompleta); Smith, A. (1981), pp. 781-788.

29 No sería pertinente abrir un argumento colateral desarrollando más este tema, el cual está muy bien tratado por Carrión, G. (2010), pp. 185-212.

30 Por ejemplo, Smith, A. (1995), pp. 392-394 y Smith, A. (1996), pp. 139-140; Smith, A. (1982c), pp. 346-347 y 491-492.

31 Smith, A. (2018), p. 93; Smith, A. (1985), pp. 223-224. 
no paseando" 32 . Es decir, Alejandro y su acción no son diferentes, son una misma realidad. Frente a eso, los lenguajes modernos se fueron haciendo cada vez más analíticos, segmentando la realidad con una artificialidad creciente ${ }^{33}$. Ejemplificó esto interpretando la palabra venit como si en su origen hubiera significado algo así como "el león viene", es decir, un hecho integrado en el que no se separaban el león, su movimiento ni su dirección; sin embargo, puesto que en el lenguaje moderno decimos: "el león se mueve hacia nosotros", separamos artificialmente el león, su movimiento y su dirección ${ }^{34}$. Por lo tanto, si comparamos el latín con el lenguaje moderno, concluiremos que mientras más analítico sea un lenguaje (y más artificial la cultura que lo produce), más separado se hallará de la síntesis natural original. Esta multiplicación artificial de las partes del lenguaje exigió la estandarización de su forma y su uso, con una sintaxis más rígida ${ }^{35}$. Mientras que las palabras en una oración latina podían variar su posición sin deformar su significado, eso no era posible en los lenguajes modernos, más articulados. El aumento de las partes, su estandarización y su rigor compositivo definieron, pues, la evolución del lenguaje ${ }^{36}$. Esta pauta fue estructuralmente la misma que Smith utilizó en su análisis histórico de la división del trabajo, que también se desarrolló a partir de una síntesis primitiva, con su libertad en la disposición secuencial de las operaciones productivas (la misma persona lo hacía todo), hacia un análisis más articulado en las sociedades comerciales, en las que el trabajo se ordenaba según una sintaxis temporal rigurosa y predeterminada (con momentos asignados a personas diferentes).

Aunque Smith dudó de la existencia del primitivo aislado, como hipótesis contra-fáctica, con la que también inició Consideraciones, representaba al ser humano sintético y completo, que hacía por sí mismo todo lo necesario para garantizar su subsistencia. En este estado imaginario, afirmó, cada individuo disfrutaría del producto íntegro de su trabajo, sin que mediasen patrones, usureros o recaudadores ${ }^{37}$. Era una ficción roussoniana que utilizó en las Lecciones sobre jurisprudencia y que en La riqueza de las naciones presentó como previa al

32 Smith, A. (2018), p. 73

33 Smith, A. (2018), pp. 72-73 y Smith, A. (1985), pp. 215-216; Rousseau, J. J. (1989), pp. 141-142; Coseriu, E. (1968), pp. 47-50; Berry, Ch. J. (1974), pp. 137-138; Land, S. K. (1986), pp. 154-157; Plank, F. (1992), pp. 41-43.

34 Smith, A. (2018), pp. 74-75 y Smith, A. (1985), pp. 216-217; Land, S. K. (1977), p. 682.

35 McKenna, S. J. (2006), p. 83.

36 Smith, A. (2018), pp. 79-92 y Smith, A. (1985), pp. 218-223; Amrozowicz, M. C. (2013), p. 157.

37 Smith, A. (2017), p. 135; Smith, A. (1982c), p. 563. 
nacimiento del lenguaje, puesto que dicho salvaje no necesitaría persuadir a nadie, marcándose con ello el hipotético comienzo de la historia de nuestra especie, que ha sido una historia de colaboración:

"En aquel estado primitivo de la sociedad en el que no existe la división del trabajo, los intercambios son escasos y cada persona se autoabastece [...] Cada hombre procura satisfacer mediante su propio trabajo las necesidades ocasionales que tenga" ${ }^{38}$.

Smith fue el principal formulador de lo que se conoce como "ley de evolución histórica de los cuatro estadios”, según la cual la sociedad progresó siguiendo la pauta caza, pastoreo, agricultura y comercio $^{39}$. La hipótesis del salvaje sintético y autosuficiente se ubicaría al comenzar la época de los cazadores, al final de la cual se iniciaría un leve proceso, imperfecto e intermitente, de distinción analítica de las ocupaciones, que Smith ejemplificó, como vimos, con un cazador que comenzó a intercambiar $\operatorname{arcos}{ }^{40}$. Aunque en el contexto de su argumento a Smith no le preocupaba fijar con precisión la posición histórica del evento, era la época de transición de la caza al pastoreo, pues quien fabricaba el arco podía obtener a cambio por él, además de presas salvajes, ganado doméstico. No era, sin embargo, la época pura del pastoreo, pues para que la fabricación de arcos pudiera ser una ocupación viable tenía que haber una demanda mínima de ellos, es decir, la caza debía aún resultar económicamente fundamental. La división de las ocupaciones surgió, por lo tanto, en la transición entre la caza y el pastoreo, es decir, después de establecido el lenguaje, el cual, según Smith, fue el único vínculo social entre los cazadores $^{41}$, quienes en la época de transición al pastoreo pudieron utilizar la fórmula básica de toda interacción, ahora ya en una versión de intercambio puramente económico, que sería: "dame un venado (o ganado) que necesito y te daré un arco que necesitas". Si los arcos ofertados fueron más efectivos que los usados previamente por el demandante, este cazaría más y la situación económica se estabilizaría. En ese momento, con todo, la síntesis todavía predominaba, pues el artesano, además de usar sus propios arcos para cazar, recolectaba la madera con la que los hacía, la trabajaba, montaba y cambiaba el

38 Smith, A. (1995), p. 381 y Smith, A. (1982c), p. 335; Smith, A. (1994), p. 355 y Smith, A. (1981), p. 276.

39 Meek, R. L. (1976), pp. 106-130; Meek, R. L. (1977), pp. 24-32; Pocock, J. G. A. (2006), pp. 280287; Rasmussen, D. C. (2008), pp. 92-101; Pauchant, Th. C. (2017), pp. 49-74.

40 Smith, A. (1994), p. 46; Smith, A. (1981), p. 27.

41 Smith, A. (1995), p. 238; Smith, A. (1996), p. 18; Smith, A. (1982c), pp. 201 y 404. 
producto finalizado, una situación de la que Smith, como Rousseau, detectó vestigios en zonas rurales, alejadas del influjo urbano ${ }^{42}$.

Como sucedió con el lenguaje (en el que los verbos impersonales se escindieron, de manera artificial según Smith, en dos partes especializadas: sujeto y verbo personal) y en conexión con ello, la especialización y la división del trabajo se fueron haciendo cada vez más intensas. Sobre todo, con el desarrollo de las comunicaciones y el crecimiento y aumento de la influencia de las ciudades, habitadas por multitudes que interactuaban intensamente entre sí, la especialización de los oficios se consolidó definitivamente ${ }^{43}$. A esta primera fase urbana de diferenciación de las ocupaciones, ya tematizada por Platón ${ }^{44}$, que alcanzó su máximo desarrollo en las ciudades renacentistas occidentales, le siguió otra, de división interna de cada oficio, que definió el mundo en el que Smith vivía.

La especialización de las ocupaciones estaba completamente consolidada en el siglo XVI, algo que se puede ver en el Libro de los oficios de Amman (1568), una buena muestra de su diversidad en una ciudad comercial del Renacimiento como Nuremberg, donde los oficios estaban separados, pero los talleres estaban poco divididos. Entre sus xilografías la fabricación de alfileres aparece como un trabajo específico, aunque el artesano hace toda la tarea salvo el empaquetado, que corresponde a una figura femenina, seguramente su esposa. En el poema de Hans Sachs que acompaña la imagen se enumeran las operaciones de las que el maestro se ocupa, que abarcan todo el proceso de fabricación ${ }^{45}$. De estos talleres renacentistas Smith dijo que "el trabajo estaba probablemente mucho menos subdividido y la maquinaria era mucho más imperfecta que en la actualidad" 46 .

Una de las cosas más conocidas de la historia de la economía es que Smith recurrió al citado oficio como introducción a las virtudes de la división del trabajo, pero no lo es tanto que en las Lecciones sobre jurisprudencia lo situó en el marco de un proceso histórico que iba desde la síntesis primitiva más completa hasta el análisis moderno más exhaustivo. Comenzó, con una imagen tan inverosímil como la del salvaje primitivo, conjeturando cómo sería el equivalente en este oficio del armero primitivo; prosiguió analizando el trabajo de

\footnotetext{
42 Smith, A. (1995), p. 119; Smith, A. (1982c), p. 86; Rousseau, J. J. (1994), pp. 75-77.

43 Smith, A. (1994), p. 49; Smith, A. (1981), p. 31.

44 Vivenza, G. (1984), pp. 179-192.

45 Amman, J. (1973), p. 84.

46 Smith, A. (1994), p. 335; Smith, A. (1981), p. 261.
} 
un maestro artesano, como el ilustrado por Amman; y acabó en el taller moderno, donde el proceso se dividía en dieciocho fases, según aparecía en la entrada "Épingle" de la Enciclopedia ${ }^{47}$.

Como decimos, Smith comenzó su análisis histórico con la conjetura del primitivo, aunque se trataba de un caso estudiadamente más complejo que la simple fabricación de un arco o unas flechas: "Supongamos que un hombre excava el mineral de la mina, lo separa y lo mezcla, lo funde, y prepara los hornos y fragua el bronce en barras, las convierte en alambre, corta este alambre, forma la cabeza, y etc., etc.; difícilmente será capaz de hacer un alfiler en el plazo de un año" ${ }^{48}$. El sorprendente resultado sería que ese alfiler se vendería, si consideramos solo el trabajo de producirlo y no tenemos en cuenta la demanda, que podría aumentar su precio, por seis libras.

El siguiente momento se corresponde con una etapa más analítica, cuando los oficios ya se habían separado. En este momento, afirmó Smith, "Si supusiéramos que la mayor parte del trabajo se le entrega hecho, y si se le da el bronce convertido en alambre, y que realiza todas las diversas operaciones requeridas para convertirlo en alfileres, no sería capaz de hacer más de 20 alfileres al día"49. En esta segunda fase, la conformación de la materia prima era un oficio diferente y el artesano se concentraba en el montaje de los alfileres, cada uno de los cuales no se podía vender por menos de un penique. Esta fase, que se corresponde con la época reflejada por Amman, es la que Smith introduce como antecedente preparatorio de las bondades de la división del trabajo en La riqueza de las naciones, donde no contempló la conjetura más radicalmente sintética de la primera fase ${ }^{50}$.

En un tercer momento, el proceso analítico dio un paso más hacia la división interna de los oficios, algo que en el mundo occidental se consolidó definitivamente en la época de Smith, quien nos introdujo, de la mano de Diderot, en los talleres más evolucionados de su época ${ }^{51}$. Las Lecciones sobre jurisprudencia nos permiten comprender mejor el proceso histórico, que condujo al paso final, según el cual el fabricante de alfileres dividía el trabajo "entre muchas manos; uno corta el alambre, otro afila un extremo para colocar la cabeza, 3 ó 4 están empleados en hacer la cabeza, uno la pone, otro hace la

47 D'Alembert, J. le R. y Diderot, D. (1755), pp. 804-808; Mankin, R. (2008), pp. 189-200.

48 Smith, A. (1995), p. 388; Smith, A. (1982c), p. 341.

49 Smith, A. (1995), p. 388; Smith, A. (1982c), p. 342.

50 Smith, A. (1994), p. 34; Smith, A. (1981), p. 14.

51 D’Alembert, J. le R. (1965), pp. 137-142. 
punta, otro lo dora y otro lo clava en el papel" ${ }^{2}$. El resultado final era que se empleaban 18 personas que fabricaban 36.000 alfileres al día (2.000 alfileres por persona), pudiéndose vender un alfiler por $1 / 24$ de penique ${ }^{53}$.

Si el trabajo del artesano de Amman se podía equiparar con el idioma latino, muy sintético y poco estructurado formalmente, gracias a su sistema de declinaciones, el trabajo minuciosamente dividido del taller moderno que presentaba la Enciclopedia era analítico, estructurándose según una secuencia temporal rigurosa, como los lenguajes modernos.

Con su análisis del lenguaje Smith no solo introdujo, pues, como veíamos al comienzo de este apartado, la idea de los efectos impremeditados del comportamiento humano, sino también algo estructuralmente más definido, a saber, la pauta evolutiva que seguían dichos efectos, incluido el trabajo y la transformación de los recursos naturales ${ }^{54}$. La historia que expuso en Consideraciones anticipó la ley del desarrollo que Herbert Spencer creyó descubrir casi un siglo después, consistente en la evolución desde una homogeneidad incoherente hacia una heterogeneidad coherente ${ }^{55}$. No queremos decir que Smith fuera un evolucionista, pero dicha ley define bastante bien la pauta que identificó cuando expuso que, al hacerse el lenguaje progresivamente más heterogéneo en sus partes, cada vez más numerosas y especializadas, tuvo que hacerse por eso mismo cada vez más coherente y sintácticamente definido. Para Smith esta pauta definía la estructura fundamental de todo proceso histórico, comenzando con el salvaje autosuficiente y acabando con la complejidad social de las ciudades modernas, conectadas entre sí a través del comercio. No pensamos que sea solo una analogía entre el lenguaje y la economía, sino, más bien, una hipótesis diacrónica global, según la cual la historia humana comenzó cuando un salvaje, comprendiendo su propia desprotección, empezó a decirle a otro, cuyo desamparo también comprendía: "dame aquello que necesito y te daré esto que necesitas".

Según Dugald Stewart, la función principal de lo que él denominó "historia conjetural", que se mostraba con toda su pureza en Consideraciones, era elucidar "por qué etapas graduales se ha efectuado la transición de los esfuer-

52 Smith, A. (1995), p. 388; Smith, A. (1982c), p. 342.

53 En La riqueza de las naciones el número total de alfileres producidos aumentaba a 48.000, pero el número de operarios era el mismo (Smith, A. -1994-, p. 35; Smith, A. -1981-, p. 15), coincidiendo con lo expuesto en la Enciclopedia.

54 Christie, J. R. R. (1987), p. 207.

55 Spencer, H. (1946), p. 325. 
zos más simples de la naturaleza inculta hasta un estado de cosas tan maravillosamente elaborado [artificial] y complejo" ${ }^{56}$, una manera de proceder que Smith extendió a La riqueza de las naciones, estableciéndose Consideraciones como su marco metodológico. Meek, analizando esta interpretación, afirmó que la historia era más conjetural en Consideraciones que en La riqueza de las naciones, pues, aunque compartían la idea de la similitud de respuestas ante situaciones similares, en La riqueza de las naciones la historia era más factual y el marco dentro del cual se organizaban los datos (la teoría de los cuatro estadios) más convincentemente realista ${ }^{57}$. No obstante, nosotros creemos que en Consideraciones Smith introdujo una hipótesis más potente para la organización de los datos, a saber, el desarrollo desde una homogeneidad incoherente hacia una heterogeneidad coherente, dentro del cual se podía integrar, como un medio de conexión con datos más empíricos, dicha teoría de los cuatro estadios.

La influencia de Consideraciones sobre el pensamiento de Smith no se agota con esto. Como vamos a ver a continuación, hay un último nivel, que creemos más relevante.

\section{EL SURGIMIENTO DE LA CONCIENCIA DIALÓGICA Y DE LA CONDUCTA TEATRALIZADA.}

La comunicación lingüística puso en marcha dos dimensiones fundamentales del proceso de socialización, las cuales alcanzaron su manifestación más compleja y sofisticada con el comportamiento ético y empresarial de los sujetos modernos: la conciencia dialógica y la teatralización de la conducta ${ }^{58}$.

\section{La conciencia dialógica.}

$\mathrm{Al}$ inicio de Consideraciones dos salvajes, carentes de lenguaje y no socializados, se encuentran y comienzan a comunicar sus necesidades mediante los objetos que las satisfacen, para ayudarse mutuamente. La comunicación tuvo que ser recíproca y el cambio de condición que se produjo tuvo que afectar a ambos salvajes. Eso lo exige la fórmula básica del lenguaje que generó el proceso de socialización, cuya función no fue solo expresiva y denotativa, sino

56 Smith, A. (1998), pp. 250-251; Smith, A. (1982b), p. 292.

57 Meek, R. L. (1976), pp. 231-235.

58 Phillipson, N. (2010), p. 165; Fleischacker, S. (2005), pp. 12-15. 
también apelativa. Esta fórmula lingüística implicaba que el hablante se pusiera en el lugar del oyente, en la medida en la que aquel debía comprender las necesidades de este; y que el hablante permitiera al oyente colocarse su lugar, en la medida en la que este también debía comprender las necesidades de aquel. Los papeles de hablante y oyente debían ser intercambiables desde el principio; por muy básicamente que eso sucediera, ambos tenían que desdoblarse en dos espectadores y dos actores sociales, dos papeles vistos desde dentro, cuyas relaciones estructurales se comprendían y compartían ${ }^{59}$. Con esto se anunciaba la estructura del comportamiento moral descrita en La teoría de los sentimientos morales, pero también la del comportamiento económico expuesta en La riqueza de las naciones ${ }^{60}$.

Smith afirmó que solo podemos asumir la conducta ajena si nos ponemos en el lugar de la persona que evaluamos, aunque sin olvidar que valoramos "la vista de usted según mi propia vista, su oído por mi oído, su razón por mi razón, su resentimiento por mi resentimiento, su amor según mi amor. No tengo ni puedo tener otra forma de juzgarlos" ${ }^{61}$. Para juzgar lo que ve otro el sujeto tiene que ponerse en su lugar y ver con sus propios ojos lo que el otro ha visto con los suyos. Es decir, tiene que reducirlo a un papel que pueda asumir, hacerse actor. Como mostró Diderot, un actor era un sujeto que, sin dejar de ser quien era, lograba ver el mundo como lo veía otro sujeto; que, sin abandonar su identidad individual, podía adoptar la de otro ${ }^{62}$. Según Smith, en nuestra vida social obramos así cotidianamente porque convertimos a los demás en papeles que tratamos de asumir.

El germen de esto estaba en el primer párrafo de Consideraciones, pues Smith también podría haber dicho que solo podemos juzgar el hambre de otro por nuestra hambre, su sed por nuestra sed o su desamparo por el nuestro, que fueron, precisamente, las experiencias fundamentales a las que se referían los primeros sustantivos en Consideraciones. Solo podemos comprender el hambre de otra persona si, por decirlo así, recreamos lo mejor posible su estómago con el nuestro, lo cual nos moverá a auxiliarla. Esta interesante idea mostraba que, en términos epistemológicos, el sujeto smithiano era una suerte de salvaje roussoniano que solo se tenía a sí mismo, si bien a través del lenguaje esta conciencia solitaria se volvió dialógica ${ }^{63}$.

59 Griswold, Ch. L. (2006), p. 23.

60 McKenna, S. J. (2006), p. 77; Dascal, M. (2006), p. 102.

61 Smith, A. (1997), p. 66; Smith, A. (1982a), p. 19.

62 Diderot, D. (1990), pp. 125-126 y 129-130.

63 Brown, V. (1994), p. 75. 
Ese fue el primero de los tres momentos (Frazer detectó cinco ${ }^{64}$ ) en el camino de la conducta teatralizada. El segundo se basa en que si hay lenguaje debe haber reciprocidad, es decir, la otra persona también debe ser un actor que, considerándome un papel a asumir, se pone en mi pellejo. El tercero consiste, finalmente, en que cada sujeto se pone en el lugar del otro cuando este se pone en el lugar de su otro, que es el sujeto de partida ${ }^{65}$, lo cual, en La teoría de los sentimientos morales, fue un momento crucial en el desarrollo del comportamiento moral:

"[...] aprobamos o desaprobamos nuestra propia conducta si sentimos que, al ponernos en el lugar de otra persona y contemplarla, por así decirlo, con sus ojos y desde su perspectiva, podemos o no podemos asumir totalmente y simpatizar con los sentimientos y móviles que la influyeron" ${ }^{66}$.

Es una teoría enrevesada, casi hegeliana. Cuando actúa, el sujeto es consciente de estar interpretando un papel que el otro tratará de asumir; con ello, para el sujeto el otro es un papel que consiste, en realidad, en verlo a sí mismo como otro papel, de manera que el sujeto ha de comportarse como un actor que asume al otro que lo asume y en el momento en el que lo asume. Por ejemplo, si tiene hambre y quiere que el otro lo ayude, ha de permitirle que, poniéndose en su lugar, comprenda la intensidad de su carencia y la honestidad de su comunicación. Para ello, tiene que anticipar cómo será asumido, viéndose a sí mismo con los ojos del otro y, así, planificar su puesta en escena. Esto era muy semiótico, dado que presuponía y desarrollaba las reflexiones de Condillac, quien mostró que el comportamiento de un salvaje, carente, en principio, de intención comunicativa, se institucionalizó y controló al influir sobre otro salvaje de manera recurrente, con lo cual el gesto espontáneo se volvió signo voluntario y la conciencia solitaria se volvió dialógica ${ }^{67}$. Esta conciencia dialógica, surgida de la fórmula elemental del lenguaje, era la que estructuraba el comportamiento y la evaluación morales en La teoría de los sentimientos morales. Se comprende, con ello, que Smith afrontara el origen del lenguaje en la tercera de sus Lecciones sobre retórica, cuyo tema central era la comunicación, y que incluyera el tema, definitivamente, en la tercera edición de La teoría de los sentimientos morales. El elemento conector era la noción de "persuasión”, que no implica necesariamente manipulación, sino tan solo deseo de

64 Frazer, M. L. (2010), p. 100.

65 Griswold, Gh. L. (1999), p. 51; Frazer, M. L. (2010), p. 100.

66 Smith, A. (1997), p. 227; Smith, A. (1982a), pp. 109-110.

67 Condillac, E. B. de (1999), pp. 153-154; Wells, G. A. (1987), pp. 8-11. 
convencer mostrando, hasta donde es posible, la intimidad de la propia situación ${ }^{68}$.

La importancia concedida a los recursos puestos en práctica para convencer explica el tipo de aproximación a la retórica en sus Lecciones sobre retórica, donde Smith abandonó la exposición tradicional, centrada en las figuras lingüísticas, para centrarse en la efectividad comunicativa ${ }^{69}$. El principio fundamental que adoptó fue la necesidad de que el hablante mostrara su condición personal de la manera más clara posible, lo que exigía tener en cuenta la situación del destinatario que debía asumir su discurso. Es decir, quien hablaba debía ponerse en el lugar de aquel a quien se dirigía, para que este pudiera ponerse, a su vez, en el lugar de aquel. Puesto que el público de dichas lecciones lo constituían sus contemporáneos escoceses, cada vez más vinculados con Inglaterra, Smith se centró adecuadamente en el uso correcto y efectivo del idioma inglés moderno, lo que requería la comprensión de su posición en la historia del lenguaje como una lengua especialmente analítica y estructurada ${ }^{70}$.

De ese entramado retórico procedía La teoría de los sentimientos morales, donde Smith escribió que deseamos mejorar nuestra situación vital para "ser observados, atendidos, considerados con simpatía, complacencia y aprobación", ganando o manteniendo, con ello, "el respeto de nuestros pares, nuestra reputación y posición en la sociedad en la que vivimos" "11. El sujeto representa su papel social de la manera más clara posible porque desea ser aprobado y, para ello, debe facilitar que el espectador pueda ponerse en su lugar, comunicando su condición y convenciendo de que es digno de aprobación. La interacción moral se articulaba, por lo tanto, en el marco de la conciencia dialógica abierta por el lenguaje.

Hablar y persuadir eran para Smith actividades complementarias, una facultad y un instinto humanos del que carecían los demás animales, como expuso al final de La teoría de los sentimientos morales:

"El deseo de ser creídos, el deseo de persuadir, de encabezar y dirigir a otras personas, parece ser uno de nuestros deseos naturales más intensos. Acaso sea el instinto sobre el que se funda la facultad del habla, la facultad característica de la naturaleza humana"72.

\footnotetext{
68 Reisman, D. A. (1976), pp. 55-59; Valihora, K. (2016), p. 418.

69 Brown, V. (2016), pp. 18-20.

70 Amrozowicz, M. C. (2016), p. 150.

71 Smith, A. (1997), pp. 124 y 382; Smith, A. (1982a), pp. 50 y 212; Swearingen, C. J. (2016), p. 170.

72 Smith, A. (1997), pp. 586-587; Smith, A. (1982a), p. 336.
} 
En el caso del lenguaje, persuadir quiere decir guiar, convencer sin violencia al otro para que nos ayude a satisfacer alguna necesidad ${ }^{73}$. En gran medida, la parte de las Lecciones sobre jurisprudencia que trataba sobre "la policía" puede considerarse una continuación de esta propuesta de La teoría de los sentimientos morales, especialmente la argumentación relacionada con la división del trabajo (núcleo duro de La riqueza de las naciones), la cual se explicaba en parte porque el ser humano, "que continuamente necesita la ayuda de los otros, debe recurrir a algunos medios para procurar su ayuda. Esto no lo hace solo mediante la zalamería y la cortesía; no espera la ayuda, a menos que pueda devolverla en beneficio de quien se la presta, o que pueda aparentar que así será" ${ }^{\prime 4}$.

El proceso humano de socialización comenzó con la fórmula fundamental de la comunicación lingüística. Esta fórmula no materializaba solo una ventaja para el hablante (actor), sino que ponía en escena algo beneficioso también para el oyente (espectador), por lo que la dimensión de persuasión resultaba crucial $^{75}$. No debía ser solo algo ventajoso, sino evidente y reflexivamente tal, de la misma manera que el comportamiento no solo debía ser moral, sino ostensiblemente (es decir, retóricamente) moral. La apariencia era, por lo tanto, algo que importaba, no solo en la moral, sino también en la economía, donde el otro debía ser convencido de que el intercambio le reportaría beneficios ${ }^{76}$.

\section{La comunicación teatralizada.}

La fórmula lingüística básica, con la condición dialógica que le subyacía, tenía que derivar hacia formas teatralizadas de persuasión, es decir, hacia la introducción del mayor número posible de elementos semióticos complementarios del lenguaje hablado ${ }^{77}$. Esto es algo que estaba presente in nuce en el intercambio original entre los dos salvajes que comenzaron a hablar, quienes, como mostraron Condillac y Rousseau, complementaron la palabra con el grito, el gesto y el movimiento de su cuerpo, usándose en las sociedades modernas complementos adicionales.

\footnotetext{
73 Schliesser, E. (2017), pp. 30-31.

74 Smith, A. (1995), p. 395; Smith, A. (1982c), p. 347.

75 McKenna, S. J. (2006), pp. 114-117.

76 Fleischacker, S. (2005), pp. 49-50.

77 Marshall, D. (1986), pp. 185-192.
} 
Creemos pertinente hacer aquí referencia brevemente a la Investigación sobre la mente bumana (1764), donde Thomas Reid trató por extenso del lenguaje, identificando en él dos dimensiones: natural (lo que hoy denominamos elementos paralingüísticos, cinésicos y proxémicos) y artificial (signos arbitrarios y convenciones del habla y la escritura). Reid creía que en la sociedad moderna la segunda dimensión se impuso a la primera, habiendo quedado esta reducida a las artes, de lo cual se lamentaba, creyendo que si durante un siglo se aboliera el lenguaje hablado y escrito "todo hombre llegaría a ser pintor, actor y orador" ${ }^{" 78}$. En relación con esto, cuando estaba definiendo sus ideas sobre el origen del lenguaje, Smith también estaba investigando sobre los sentidos externos, alabando la comparación de Berkeley entre lenguaje de la visión y el de la palabra ${ }^{79}$, lo que mostraba su interés tanto en los lenguajes "naturales" como en los artificiales, respecto a lo cual creía, a diferencia de Reid, que en nuestra cotidianeidad moderna no se había producido la reducción de lo natural a lo artificial, sino una compleja integración de ambos en un empeño comunicativo común ${ }^{80}$.

En su biografía de Smith, Stewart afirmó que a este le interesaron los métodos de composición escénica y la historia del teatro por "su conexión con los principios generales de la mente humana" ${ }^{81}$. De hecho, entre los ejemplos usados en La teoría de los sentimientos morales para ilustrar nuestro comportamiento cotidiano muchos provienen del teatro, que proporcionó a esta obra ejemplos y tipos morales ${ }^{82}$. Dada la importancia del atrezo como complemento de los personajes, nos parece evidente que Smith no pensaba que nuestro lenguaje hubiera abandonado los elementos no verbales, que se habrían refugiado en el arte, sino que para él la comunicación cotidiana era un todo complejo e integrado, como quedó claro cuando en clase, refiriéndose a los conceptos fundamentales de su retórica de la comunicación, dijo que eran "tan aplicables a la conversación y a la conducta como a la escritura" ${ }^{83}$.

Smith disentía de Reid, además, en relación con la naturalidad de los lenguajes que se habían refugiado en el arte, pues, según su hipótesis del desa-

\footnotetext{
78 Reid, Th. (2004), p. 115.

79 Smith, A. (1998), pp. 158-160; Smith, A. (1982c), pp. 156-158.

80 Rothschild, E. (2010), p. 27.

81 Smith, A. (1998), pp. 264-273; Smith, A. (1982b), p. 305.

82 Griswold, Ch. L. (1999), p. 65; Buchan, J. (2007), p. 52.

83 "[...] equally applicable to conversation and behaviour as writing"; Smith, A. (1985), p. 55. Traducción nuestra.
} 
rrollo, el lenguaje era cada vez más artificial ${ }^{84}$. Las artes imitativas eran para él un artificio cultural integrado, a través del diseño, en una cotidianeidad cada vez más elaborada, algo debido a la transformación histórica de la sociedad según la pauta caza, pastoreo, agricultura y comercio, con el aumento de la propiedad privada y de las interacciones entre personas, aunque el esquema dialógico instaurado con el surgimiento lenguaje se mantenía como núcleo inalterado. Los dos salvajes iniciales, dentro del escenario del bosque, contaban con los movimientos de su cuerpo, los gestos de su rostro y los sonidos que podían emitir para hacer creíble ante el otro su estado carencial, pero cuando la sociedad evolucionó hacia el comercio los recursos se volvieron más complejos y artificiales ${ }^{85}$, un mundo de lujosas habitaciones georgianas decoradas primorosamente con telas, porcelanas, cristalerías y muebles en el estilo Adam, pobladas por una fauna numerosa y diversa de vestidos a la moda, complementados con todo tipo de bagatelas y obras de arte. Los recursos comunicativos que conformaban el atrezo cotidiano de los contemporáneos de Smith eran más numerosos y complejos de lo que se había visto nunca, conformando un ambiente que el ser humano se había dado a sí mismo y con el que podía comunicar matices antes insospechados. Con todo, su sentido dialógico seguía siendo permitir que los otros comprendieran lo mejor posible nuestro comportamiento para suscitar su simpatía y, con ella, su disponibilidad a ayudarnos. Smith lo dejó claro en varios lugares de La teoría de los sentimientos morales, donde afirmó que nuestro afán de enriquecimiento no tenía una base corporal sino mental, a saber, el deseo de lograr la simpatía de nuestros iguales, por una parte, y el conocimiento de que "los seres humanos están dispuestos a simpatizar más completamente con nuestra dicha que con nuestro pesar", por otra ${ }^{86}$.

Para muchos analistas la simpatía es, tal vez, el elemento fundamental en la ética de Smith, proveniente de Hume ${ }^{87}$. Sin embargo, nosotros no hemos profundizado en ella porque creemos que la simpatía, pese a su importancia innegable, fue para Smith algo derivado, una de las consecuencias no planificadas de la dialógica de la interacción lingüística, que nos obliga a ponernos en el lugar del otro y al otro a ponerse en el nuestro. Este "ponerse en lugar de" es previo a la respuesta simpática y exige que el espectador reconstruya la si-

84 Christie, J. R. R. (1987), p. 227; McKenna, S. J. (2006), pp. 98-104; Labio, C. (2016), p. 109; Swearingen, C. J. (2016), p. 160.

85 Labio, C. (2016), pp. 116-117.

86 Smith, A. (1997), pp. 123 y 382; Smith, A. (1982a), pp. 50 y 213.

87 Turco, L. (2010), pp. 146-150; Sayre-McCord, G. (2015), pp. 211-220. 
tuación del actor. Esta reconstrucción exige el esfuerzo de la imaginación, la cual se basa en la manera en la que, a través del lenguaje, ella misma ha ido estructurando la realidad. Sin esto no podría surgir la simpatía.

Aunque el núcleo dialógico era el mismo, la situación económica era, por supuesto, diferente, pues el deseo de comunicar el estatus, cada vez más intenso con el desarrollo social, tuvo claras consecuencias sobre la complejidad y la riqueza de las naciones, aunque, a su vez, fue la riqueza creciente la que permitió que esta manera de comunicar el estatus se generalizara. En este contexto apareció por primera vez la metáfora de la mano invisible en su sentido smithiano (la expresión apareció anteriormente en su Historia de la astronomía con un significado diferente), para explicar que, cuando los ricos modernos recurrían, para marcar su estatus, a los recursos escenográficos que la artificiosa y superflua sociedad comercial ponía a su servicio, para favorecer con ello que los demás, asumiendo sus papeles, pudieran ver el mundo con sus ojos, gastaban en ello gran parte de su dinero, con lo que se producía una redistribución de la riqueza, como si "una mano invisible" los condujera "a realizar casi la misma distribución de las cosas necesarias para la vida que habría tenido lugar si la tierra hubiese sido dividida en proporciones iguales entre todos sus habitantes, y así sin pretenderlo, sin saberlo, promueven el interés de la sociedad" $"$. El gasto que debían asumir para teatralizar dialógicamente sus vidas distribuía, por lo tanto, parte del bienestar que la sociedad comercial generaba ${ }^{89}$. Aunque en La riqueza de las naciones Smith valoró esto de una manera diferente, considerando como preferibles la parsimonia y la moderación en el gasto de los beneficios del capital, a través de la distinción de dos tipos de gastos superfluos (en objetos consumibles -por ejemplo, una fiesta- y perdurables -por ejemplo, un edificio) llegó a unas conclusiones parecidas:

"[...] si el gasto de la fiesta se hubiese invertido en dar empleo a albañiles, carpinteros, tapiceros, mecánicos, etc., una cantidad de provisiones de igual valor habría sido distribuida entre un número mayor de personas"90.

Con ello, la dialógica del lenguaje y su teatralización, cuya emergencia Smith expuso en Consideraciones, acababa vinculándose, a través del desarrollo comunicativo de la conducta en La teoría de los sentimientos morales, con la dimensión económica en La riqueza de las naciones ${ }^{91}$.

88 Smith, A. (1997), p. 333; Smith, A. (1982a), pp. 184-185.

89 Debes, R. (2016), pp. 193-194.

90 Smith, A. (1994), p. 447; Smith, A. (1981), pp. 348-349.

91 Griswold, Ch. L. (2006), pp. 45-46. 
La complejidad creciente del proceso histórico, desde el encuentro conjetural de los dos salvajes hasta la sociedad comercial ilustrada, se desarrolló a través de la ley de los cuatro estadios, resultando especialmente importante la última fase, la transformación de la sociedad agrícola en comercial. En las sociedades puramente agrícolas el excedente productivo era gastado en mantener a una cohorte de siervos y seguidores, pero en las comerciales ese gasto se centraba en el mundo de objetos que rodeaban al rico. Aunque Smith lo definió como un gasto en "la más pueril, despreciable y sórdida de todas las vanidades", estas "baratijas y fruslerías propias para servir más como juguetes de niños que como objetivos serios de hombres" eran parte del atrezo que guiaba la observación y estima hacia el rico, el resultado de una exigencia, impuesta por nuestra condición dialógica, que multiplicaba constantemente la fórmula básica del lenguaje ${ }^{92}$. Por eso, también en La riqueza de las naciones Smith tuvo que acabar reconociendo que tales banalidades producían "una revolución de la máxima importancia” en el bienestar público, aunque, paradójicamente, generada otra vez por "personas que no tenían la menor intención de servir al público" ${ }^{\prime 3}$. El egoísmo loco, pero explicable, de la vanidad desatada en el sistema de estatus de la sociedad comercial, era, pues, uno de los pilares fundamentales de la posible generalización de la felicidad en el mundo moderno.

Todo esto surgió del lenguaje. Cuando los dos salvajes hipotéticos se encontraron se comunicaron, para obtener ayuda, sus necesidades básicas a través de la denotación de los objetos que las satisfacían. Con ello surgió la mente dialógica y la actuación teatralizada, pues cada hablante debía ponerse en el lugar del oyente y persuadirle, al mismo tiempo, de que, poniéndose en su lugar, lo ayudase. De esto surgió la dialógica del comportamiento y evaluación morales en La teoría de los sentimientos morales. Con el proceso civilizatorio, que evolucionó desde la caza hasta el comercio, tanto el lenguaje como la cultura se fueron volviendo más analíticos y artificiales, aprovechando la segunda la multiplicación de la cantidad y diversidad de objetos de consumo privado que la economía proporcionaba. Con esto, nuestra naturaleza necesitada se fue encauzando hacia lo superfluo y la persuasión retórica se fue haciendo cada vez más compleja y eficiente, sobre todo en el nivel de su teatralización, generando, a través de la ley de las consecuencias no planificadas de nuestros actos, un proceso de distribución de la riqueza hacia abajo, dando Smith con ello una

92 Smith, A. (1994), pp. 526 y 528; Smith, A. (1981), pp. 419 y 421.

93 Smith, A. (1994), p. 529; Smith, A. (1981), p. 422. 
brillante explicación de las bases comportamentales y económicas de la ostentación a través del consumo.

\section{CONCLUSIÓN.}

Para Smith la simpatía, como hemos visto, no es una imitación que provenga de un contagio sentimental, sino un posicionamiento imaginativo en el lugar del otro ${ }^{94}$. Al exigirnos, con ello, comprenderlo mejor, el cerebro humano abre la posibilidad de experiencias nuevas en el mundo natural, como la belleza, el desinterés o el amor de seres como Margaret, la madre de Adam Smith, la cual, como afirmó Marçal, a diferencia del cervecero o del carnicero, no se relacionaba con su hijo por interés, sino por simpatía. Marçal identificó la aproximación de Smith a la economía a través del cervecero o del carnicero como algo masculino y, como alternativa, se preguntaba: “¿era así realmente? ¿Quién le preparaba, a la hora de la verdad, ese filete a Adam Smith?”95. La conclusión de su conocida observación de que su madre no le hacía la cena por interés egoísta, sino por amor, es digna de ser tenida en cuenta: "Hemos de decir adiós al hombre económico y construir una sociedad que dé cabida a una concepción más amplia de integradora de lo humano" ${ }^{\text {" }}$.

Si leemos a Smith a partir de su teoría del lenguaje, él mismo nos proporciona medios para desarrollar la sugerencia de Marçal, pues desde el lenguaje la lectura mecanicista que ella hace del newtonianismo de Smith no se sostiene. La ley de las consecuencias impremeditadas de nuestros actos, anticipada en Consideraciones, no afirma que nuestro comportamiento esté determinado, sino que sus consecuencias se nos suelen escapar, pese a lo cual seguimos decidiendo cómo actuar. Aunque el sistema económico desarrollado por Smith fuera legaliforme, según un modelo constructivo newtoniano (de lo que nosotros, personalmente, no estamos muy seguros), para él no dejaría por eso de ser un producto de nuestras decisiones y, por mucho que no podamos controlar sus consecuencias, no es naturaleza sino cultura. Esta es la base de la relectura que hizo Roberts de la obra de Smith cuando, a partir de una reflexión sobre el surgimiento de nuevas palabras (sin citar, no obstante, Consideraciones, aunque es significativa la naturaleza lingüística de su ejemplo),

94 Chandler, J. (2016), p. 137; Frazer, M. L. (2010), p. 97.

95 Marçal, K. (2017), p. 27.

96 Marçal, K. (2017), p. 196. 
concluyó que somos nosotros mismos, a través del impacto acumulativo de una altísima serie de opciones personales, los que decidimos "qué es aceptable y qué no lo es, lo que es virtuoso y lo que no. Decidimos acerca de todas estas cosas -los fundamentos de la moral y de la civilización- del mismo modo que decidimos acerca de qué constituye un inglés aceptable" ${ }^{97}$. Con esto se da una respuesta moderada a la objeción de Marçal, pues al menos se muestra que el egoísmo interesado no define el único modelo aceptable de nuestro comportamiento económico. Smith, de hecho, pensaba que era uno de los posibles, pero no el único, ni siquiera el más deseable, como dejo claro en La teoría de los sentimientos morales cuando planteó la posibilidad de que la ayuda mutua también procediera del "amor, la gratitud, la amistad y la estima". Es más, pensaba que, en realidad solo así "la sociedad florece y es feliz"98.

Las consecuencias impremeditadas de nuestras acciones no son una causa sino un efecto, son un resultado y no un destino, el producto de un lenguaje que cada vez se aleja más de la naturaleza y depende más de nosotros, no de un conjunto de leyes físicas inmutables. Tal vez la naturaleza nos imponga, como dijo Smith, una piel delicada que nos exige cubrirnos, pero el deseo de vestir un chaleco de seda teñida de celeste con bordados de plata de motivos vegetales es una creación de nuestra industria y nuestra moda, que tratan de satisfacer deseos dependientes de nuestra condición de animal social semiótico e inacabado. Quizá nuestro destino sea vestirnos, pero nosotros elegimos cómo lo hacemos. Del mismo modo, puede que nuestro destino sea ser sociales, pero podemos elegir si lo somos según el modelo de Mandeville o según una simpatía más pura que pueda surgir de la dialógica del lenguaje. No es nuestro destino que el cervecero nos proporcione cerveza por egoísmo y no por simpatía, sino el resultado coyuntural de decisiones humanas que se basan en nuestra naturaleza lingüística, y el lenguaje, como se sabe, siempre puede decir otras cosas.

Concluyendo, la dialógica del lenguaje tiene dos consecuencias que trascienden el estado carencial originario del que surgió ${ }^{99}$ :

1) Puesto que nos pone en el lugar del otro, nos exige dotarlo de humanidad, aunque manteniendo su alteridad.

97 Roberts, R. (2015), pp. 209-210.

98 Smith, A. (1997), p. 185; Smith, A. (1982a), p. 85.

99 Griswold, Ch. L. (1999), pp. 106-108. 
2) Puesto que exige que nos veamos con los ojos del otro, hace que nos distanciemos de nosotros mismos, permitiéndonos controlar mejor nuestra existencia personal, es decir, haciéndonos más libres.

Se trata de dos resultados innegables de la fórmula lingüística, cuyo surgimiento expuso Smith en Consideraciones. Puesto que el que habla persuade, no imponiéndose por la fuerza, sino abriéndose al otro y distanciándose de su propia identidad, abre la posibilidad de la belleza, el amor y el respeto como cosas valiosas en sí mismas. Podemos persuadir a los demás, o dejarnos persuadir por ellos, no solo de que nos proporcionen comida, bebida o vivienda, cerveza o carne, sino de que valdría la pena que conjuntamente construyéramos un mundo en el que todos viviéramos mejor. Esto sería también una consecuencia imprevista de nuestra condición semiótica, la cual nos impediría afirmar que el capitalismo salvaje (cuyos economistas se han apropiado de Smith como santo patrón ${ }^{100}$ ) sea la última palabra humana.

Hace unos años, Meek afirmó que la época centrada en la teoría económica de Smith estaba empezando a ser superada porque el ser humano estaba comenzando a dominar la máquina que, hasta entonces, había controlado su destino económico ${ }^{101}$. Nosotros creemos que esa posibilidad de control creativo es intrínseca al núcleo más profundo del pensamiento de Smith, al menos si lo enraizamos en Consideraciones. El capitalismo salvaje, que generó esa imagen de una máquina inmisericorde cuyo origen y crisis detectó Meek, es un producto, a la vez, de nuestra naturaleza necesitada y de nuestra condición semiótica, pero esta misma condición, a través de la simpatía generada por la conciencia dialógica, permite concebir que las cosas siempre podrían ser de otra manera, afirmando para empezar, por ejemplo, que el "comercio [...] debería ser entre las naciones [...] como entre los individuos [...] un lazo de unión y amistad"102.

100 Erróneamente según Cox, H. (2016), pp. 142-175.

101 Meek, R. L. (1977), p. 187.

102 Smith, A. (1994), p. 564; Smith, A. (1981), p. 493. 


\section{BIBLIOGRAFÍA}

Amman, Jost (1973), The Book of Trades, Dover, Nueva York.

Amrozowics, Michael C. (2016), "Adam Smith: History and Poetics”, en Berry, Christopher J., Paganelli, Maria Pia, y Smith, Craig (eds.), The Oxford Handbook of Adam Smith, Oxford University Press, Oxford, pp. 143-158.

Berry, Christopher J. (1974), "Adam Smith's Considerations on Language", Journal of the History of Ideas, vol. 35, pp. 130-138.

Brown, Vivienne (1994), Adam Smith's Discourse, Routledge, Londres.

Brown, Vivienne (2016), "The Lectures on Rhetoric and Belles Lettres", en Hanley, Ryan Patrick (ed.), Adam Smith. His Life, Thought, and Legacy, Princeton University Press, Princeton, pp. 17-32.

Buchan, James (2007), Adam Smith and the Pursuit of Perfect Liberty, Profile Books, Londres.

Carrión, Gonzalo (2010), "Imaginación, metáfora y gnoseología en el pensamiento de Adam Smith", Revista Empresa y Humanismo, vol. XIII, n ${ }^{\circ}$, pp. 185-212.

Chandler, James (2016) "Adam Smith as critic", en Berry, Christopher J., Paganelli, Maria Pia, y Smith, Craig (eds.), The Oxford Handbook of Adam Smith, Oxford University Press, Oxford, pp. 126-142.

Christie, John R. R. (1987), "Adam Smith's Metaphysics of Language", en Benjamin, Andrew E.; Cantor, Geoffrey N. y Christie, John R.R. (eds.), The Figural and the Literal, Manchester University Press, Manchester, pp. 202229.

Condillac, Étienne Bonnot de [1746 (1999)], Ensayo sobre el Origen de los Conocimientos Humanos, Tecnos, Madrid.

Conlin, Jonathan (2016), Adam Smith, Reaktion Books, Londres.

Coseriu, Eugenio (1968), "Adam Smith und die Anfänge der Sprachtypologie”, en Brekle, Herbert E. y Lipka, Leonard (eds.), Wortbildung, Syntax, und Morphologie, Mouton, La Haya, pp. 46-54.

Cox, Harvey (2016), The Market as God, Harvard University Press, Cambridge/Massachusetts. 
D’Alembert, Jean le Ron y Diderot, Denis (eds.) (1755), Encyclopédie ou Dictionnaire Raisonné des Sciences, des Arts et des Métiers, Tome V, Le Breton/Durand/Briasson/Michel-Antoine David, París.

D'Alembert, Jean le Rond [1751 (1965)], Discours Préliminaire de l'Encyclopédie, Gonthier, París.

Dascal, Marcelo (2006), "Adam Smith's Theory of Language”, en Haakonssen, Knud (ed.), The Cambridge Companion to Adam Smith, Cambridge University Press, Cambridge, pp. 79-111.

Debes, Remy (2016), "Adam Smith and the Sympathetic Imagination", en Hanley, Ryan Patrick (ed.), Adam Smith. His Life, Thought, and Legacy. Princeton University Press, Princeton, pp. 192-207.

Diderot, Denis (1990), Paradoja del Comediante y Otros Ensayos, Mondadori, Madrid.

Fleischacker, Samuel (2005), On Adam Smith's Wealth of Nations, Princeton University Press, Princeton.

Frazer, Michael L. (2010), The Enlightenment of Sympathy, Oxford University Press, Oxford.

Griswold, Charles L. (1999), Adam Smith and the Virtues of Enlightenment, Cambridge University Press, Cambridge.

Griswold, Charles L. (2006), "Imagination: Moral, Science, and Arts", en Haakonssen, Knud (ed.), The Cambridge Companion to Adam Smith, Cambridge University Press, Cambridge, pp. 22-56.

Hume, David [1748 (2015)], Investigación sobre el conocimiento bumano, Alianza, Madrid.

Keller, Rudi (1994), On Language Change. The Invisible Hand in Language, Routledge, Abingdon.

Labio, Catherine (2016), "Adam Smith's Aesthetics”, en Berry, Christopher J.; Paganelli, Maria Pia y Smith, Craig (eds.), The Oxford Handbook of Adam Smith, Oxford University Press, Oxford, pp. 105-125.

Land, Stephen K. (1977), “Adam Smith's 'Considerations Concerning the First Formation of Languages", Fournal of the History of Ideas, vol. 38, pp. 677-690. 
Land, Stephen K. (1986), The Philosophy of Language in Britain, Ams Press, Nueva York.

Mankin, Robert (2008), "Pins and Needles: Adam Smith and the Sources of the Encyclopédie", Adam Smith Review, vol. 4, pp. 181-206.

Marçal, Katrine (2016), ¿Quién bacía la Cena a Adam Smith?, Debate, Barcelona.

Marshall, David (1986), The Figure of the Theater. Shaftesbury, Defoe, Adam Smith, and George Eliot, Columbia University Press, Nueva York.

McKenna, Stephen J. (2006), Adam Smith. The Rhetoric of Propriety, State University of New York Press, Albany/Nueva York.

McKenna, Stephen J. (2016), "Adam Smith and Rhetoric”, en Hanley, Ryan Patrick (ed.), Adam Smith. His Life, Thought, and Legacy, Princeton University Press, Princeton, pp. 387-404.

Meek, Ronald L. (1976), Social Science and the Ignoble Savage, Cambridge University Press, Cambridge.

Meek, Ronald L. (1977), Smith, Marx, and After, Chapman and Hall, Londres.

Montes Lira, Leónidas (2009), "La influencia de Newton en Adam Smith”, Anuario Filosófico, vol. XLII, nº 1, pp. 137-158.

Otteson, James R. (2002a), Adam Smith's Marketplace of Life, Cambridge University Press, Cambridge.

Otteson, James R. (2002b), “Adam Smith's First Market: The Development of Language", History of Philosophy Quarterly, vol. 19, n 1, pp. 65-86.

Otteson, James R. (2013), Adam Smith, Bloomsbury Academic, Nueva York.

Pauchant, Thierry C. (2017), "Adam Smith's Four Stages Theory of Socio-cultural Evolution: New Insights from His 1749 Lecture", The Adam Smith Review, vol. 9, pp. 49-74.

Phillipson, Nicholas (2010), Adam Smith. An Enlightened Life, Penguin, Londres.

Plank, Frans (1992), “Adam Smith: Grammatical Economist”, en Jones, Peter y Skinner, Andrew Stewart (eds.), Adam Smith Reviewed, Edinburgh University Press, Edimburgo, pp. 21-55. 
Pocock, J.G.A. (2006), "Adam Smith and History", en Haakonssen, Knud (ed.), The Cambridge Companion to Adam Smith, Cambridge University Press, Cambridge, pp. 270-287.

Rasmussen, Dennis C. (2008), The Problems and Promise of Commercial Society, The Pennsylvania State University Press, University Park/Pensilvania.

Reid, Thomas [1764 (2004)], Investigación sobre la Mente Humana, Trotta, Madrid.

Reisman, David Alexander (1976), Adam Smith's Sociological Economics, Croom Helm, Londres.

Roberts, Russ (2015), Cómo Adam Smith puede cambiar tu vida, Antoni Bosch, Barcelona.

Rousseau, Jean-Jacques (1989), Discurso sobre el Origen y los Fundamentos de la Desigualdad entre los Hombres y otros Escritos, Madrid: Tecnos.

Rousseau, Jean-Jacques [1758 (1994)], Carta a D'Alembert, Tecnos, Madrid.

Rothschild, Emma (2010), "The Theory of Moral Sentiments and the Inner Life”, The Adam Smith Review, vol. 5, pp. 25-36.

Sayre-McCord, Geoffrey (2015), "Hume and Smith on Sympathy, Approbation, and Moral Judgment", en Schliesser, Eric (ed.), Sympathy. A History, Oxford University Press, Oxford, pp. 208-246.

Schliesser, Eric (2017), Adam Smith. Systematic Philosopher and Public Thinker, Oxford University Press, Oxford.

Smith, Adam [1776 (1981)], An Inquiry into the Nature and Causes of the Wealth of Nations, Liberty Press, Indianapolis.

Smith, Adam [1759 (1982a)], The Theory of Moral Sentiments, Liberty Press, Indianapolis.

Smith, Adam (1982b), Essays on Philosophical Subjects, Liberty Press, Indianapolis.

Smith, Adam (1982c), Lectures on Furisprudence, Liberty Press, Indianapolis.

Smith, Adam (1985), Lectures on Rhetoric and Belles Lettres, Liberty Press, Indianapolis. 
Smith, Adam [1776 (1994)], La Riqueza de las Naciones, Alianza, Madrid.

Smith, Adam (1995), Lecciones sobre furisprudencia, Comares, Granada.

Smith, Adam (1996), Lecciones de furisprudencia, Centro de Estudios Constitucionales, Madrid. Madrid.

Smith, Adam [1759 (1997)], La Teoría de los Sentimientos Morales, Alianza,

Smith, Adam (1998), Ensayos Filosóficos, Pirámide, Madrid.

Smith, A. (2017), Escritos preliminares de la Riqueza de las naciones y Consideraciones sobre la primera formación de las lenguas, Biblioteca Nueva, Madrid.

Smith, Adam (2018), Consideraciones sobre la formación original de los lenguajes, KRK, Oviedo.

Spencer, Herbert (1946), First Principles, Watts and Co., Londres.

Swearingen, C. Jan (2016), "Adam Smith on Language and Rhetoric: The Ethics of Style, Character, and Propriety", en Berry, Christopher J.; Paganelli, Maria Pia y Smith, Craig (eds.), The Oxford Handbook of Adam Smith, Oxford University Press, Oxford, pp. 159-175.

Turco, Luigi (2010), "Moral Sense and the Foundation of Morals", en Broadie, Alexander (ed.), The Cambridge Companion to the Scottish Enlightenment, Cambridge University Press, Cambridge, pp. 136-156.

Valihora, Karen (2016), “Adam Smith's Narrative Line”, en Hanley, Ryan Patrick (ed.), Adam Smith. His Life, Thought, and Legacy, Princeton University Press, Princeton, pp. 405-421.

Vivenza, Gloria (1984), Adam Smith e la Cultura Classica, Il Pensiero Economico Moderno, Pisa.

Wells, George Alberts (1987), The Origin of Language. Aspects of the Discussion from Condillac to Wundt, Open Court, La Salle/Illinois. 